\title{
Hem12, an enzyme of heme biosynthesis pathway, is monoubiquitinated by Rsp5 ubiquitin ligase in yeast cells*
}

\author{
Anna Chelstowska, Zaneta Jastrzebska, Joanna Kaminska, Anna Sadurska, \\ Danuta Plochocka, Joanna Rytka and Teresa Zoladek ${ }^{\bowtie}$ \\ Institute of Biochemistry and Biophysics Polish Academy of Sciences, Warsaw, Poland
}

\begin{abstract}
Heme biosynthesis pathway is conserved in yeast and humans and hem 12 yeast mutants mimic porphyria cutanea tarda (PCT), a hereditary human disease caused by mutations in the UROD gene. Even though mutations in other genes also affect UROD activity and predispose to sporadic PCT, the regulation of UROD is unknown. Here, we used yeast as a model to study regulation of Hem 12 by ubiquitination and involvement of Rsp5 ubiquitin ligase in this process. We found that Hem12 is monoubiquitinated in vivo by Rsp5. Hem 12 contains three conserved lysine residues located on the protein surface that can potentially be ubiquitinated and lysine $\mathrm{K} 8$ is close to the 36-LPEY-39 (PY) motif which binds WW domains of the Rsp5 ligase. The hem 12-K8A mutation results in a defect in cell growth on a glycerol medium at $38^{\circ} \mathrm{C}$ but it does not affect the level of Hem12. The hem 12-L36A,P37A mutations which destroy the PY motif result in a more profound growth defect on both, glycerol and glucose-containing media. However, after several passages on the glucose medium, the hem 12-L36A,P37A cells adapt to the growth medium owing to higher expression of hem12-L36A,P37A gene and higher stability of the mutant Hem12-L36A,P37A protein. The Hem12 protein is downregulated upon heat stress in a Rsp5independent way. Thus, Rsp5-dependent Hem12 monoubiquitination is important for its functioning, but not required for its degradation. Since Rsp5 has homologs among the Nedd4 family of ubiquitin ligases in humans, a similar regulation by ubiquitination might be also important for functioning of the human UROD.
\end{abstract}

Key words: yeast; heme biosynthesis; Hem12; ubiquitination; Rsp5 ligase; protein degradation

Received: 21 April, 2014; revised: 31 July, 2015; accepted: 03 August, 2015; available on-line: 28 August, 2015

\section{INTRODUCTION}

Rsp5 is a unique yeast member of the Nedd4 family of ubiquitin ligases which have a common C2-WWHECT modular structure with the lipid-binding domain C2, protein-binding domains WW and catalytic HECT domain (Kaliszewski \& Zoladek, 2008). The Rsp5 ligase monoubiquitinates and polyubiquitinates substrates with K63-linked ubiquitin chains in vivo and in vitro (Kee et al., 2006). Some Rsp5 substrates are then transported via endocytosis to the vacuole for degradation (Lauwers et al., 2010), others are proteolytically processed (Hoppe et al., 2000) or have reduced activity (Novoselova et al., 2013). Rsp5 ubiquitinates a transcriptional activator, Spt23, thereby promoting its release from the endoplasmic re- ticulum, also affecting its nuclear transport and transcriptional activation of the OLE1 gene encoding a desaturase of fatty acids. This is an essential function since $r s p 5 \Delta$ strain cannot grow unless cells bear a plasmid encoding a constitutively active variant Spt231-689 (Hoppe et al., 2000). Rsp5 is also involved in proteasomal degradation of Rpb1, the largest subunit of the RNA polymerase II (Harreman et al., 2009). This protein is ubiquitinated in a two-step mechanism in which Rsp5 first adds monoubiquitin and then a second ubiquitin ligase produces a K48-linked polyubiquitin chain, which triggers proteasomal proteolysis (Harreman et al., 2009). The rsp5 mutants are hypersensitive to various toxic compounds, including hydrogen peroxide which causes oxidative stress (Hoshikawa et al., 2003).

One of the proteins which binds to and is ubiquitinated by Rsp5 in vitro is Hem12 (Hesselberth et al., 2006; Gupta et al., 2007), a cytoplasmic uroporphyrinogen III decarboxylase (UROD), the fifth enzyme in the heme biosynthesis pathway. The HEM12 gene is essential in yeast and hem $12 \Delta$ cells do not grow unless the medium is supplemented with ergosterol and unsaturated fatty acids since Erg5 sterol desaturase and Ole1 fatty acid desaturase are hemoproteins. The hem $12 \Delta$ mutant cells supplemented with those lipids are still respiratory-deficient and do not utilize glycerol, since they lack mitochondrial cytochromes. The heme biosynthesis pathway is conserved in humans and mutations in the human UROD gene resulting in UROD dysfunction cause a hereditary disease, porphyria cutanea tarda (PCT) (Frank \& Poblete-Gutierrez, 2010), in which accumulation of porphyrins, phototoxic heme precursors, results in liver and skin damage (Mendez et al., 2012). Some mutations in the UROD gene result in lower activity and instability of UROD. Mutations in other genes may affect UROD activity and predispose to sporadic PCT by unknown mechanisms (Garey et al., 1993). In yeast cells, point mutations in the HEM12 gene also lead to the accumulation of porphyrins (Zoladek et al., 1996) and the bem12 mutants mimic PCT (Kurlandzka et al., 1988). Yeast strains with mutations in unknown genes that affect expression of HEM12 have also been isolated, resembling the situation in humans (Zoladek et al., 1995). Moreover, UROD was recently identified as a potential anticancer

e-mail: teresa@ibb.waw.p

*Preliminary report was presented at a poster session of the 26th International Conference on Yeast Genetics and Molecular Biology held at the Frankfurt, Germany, August 29th-September 3rd, 2013. Abbreviations: ALA, 5-aminolevulinic acid; PCT, porphyria cutanea tarda; PY, LPEY motif; UROD, uroporphyrinogen III decarboxylase 
target and UROD inhibitor combined effectively with radiation and other anticancer drugs (Yip et al., 2014).

Heme, as the prosthetic group of numerous hemoproteins, is crucial in electron transport in the mitochondrial oxidative chain, in several metabolic pathways, and in the defense against reactive oxygen species (Heinemann et al., 2008). Heme is essential for life but in excess it can be toxic to cells, therefore a crucial aspect of heme biosynthesis is its regulation. In mammals, the activity of 5-aminolevulinic acid (ALA) synthase, catalyzing the first step of heme biosynthesis pathway, is rate-limiting (Panda et al., 2002). Recently, a negative feedback regulation of heme homeostasis has been identified with Rev-erb $\alpha$ heme receptor interacting with the NCoR protein to repress its target genes in human cells (Wu et al., 2009). Yeast growth on glycerol medium results in a $\sim 3$-fold induction of heme biosynthesis compared with glucosegrown cells (Diflumeri et al., 1993). In yeast cells, ALA synthesis is not rate-limiting since ALA is present in excess; instead the rate-limiting are other enzymes, including Hem12 UROD (Hoffman et al., 2003). Hem12 protein could potentially be regulated post-translationally since it has been found to interact with WW domains of the Rsp5 ubiquitn ligase (Hesselberth et al., 2006) and be ubiquitinated in vitro (Gupta et al., 2007). Here, we analyzed a possible regulatory mechanism, ubiquitination of Hem12 protein in vivo, its dependence on the Rsp5 ubiquitin ligase, and significance for yeast physiology.

\section{MATERIALS AND METHODS}

Strains, media and growth conditions. The Saccharomyces cerevisiae strains used were MHY501 (Chen et al., 1993), PC10 rsp5-1 isogenic to MHY501 (P. Cholbinski IBB PAS), rsp5 (Hoppe et al., 2000), BY4741 and its derivative Y23983 HEM12/hem124::kanMX4 (Euroscarf). The hem $12 \Delta$ mutants bearing plasmids with hem12$K 8 A$ or hem12-L $36 A, P 37 A$ allele were obtained by sporulation and tetrad dissection of respective transformants of a HEM12/ hem12 $\Delta$ diploid strain.

Yeast were grown in YPD with $2 \%$ glucose, YPGly with $2 \%$ glycerol, SD or SC with $2 \%$ glucose (Sherman, 2002). YPD+G418 was used to select strains resistant to kanamycin, and sporulation medium (Sherman, 2002) for spore production. To monitor the effect of elevated temperature on the level of wild type and mutant HAHem12, the respective yeast cells were grown at $28^{\circ} \mathrm{C}$ in SC-leu medium, transferred to YPD to $\mathrm{OD}_{600} \sim 0.3$, grown for two generations and shifted to $38^{\circ} \mathrm{C}$ for 2 or 4 hours. For cycloheximide-chase analysis of Hem 12 degradation, yeast were grown in YPD medium to the logarithmic phase, and $0.5 \mathrm{ml}$ aliquots were removed for immunoblotting at indicated times following the addition of $500 \mu \mathrm{g} / \mathrm{ml}$ cycloheximide (Sigma).

Plasmids and plasmid construction. Plasmid YEpHIS-UBI was used (Stawiecka-Mirota et al., 2007). Plasmid pBS-HA-HEM12 was constructed by transfer of NotI digested DNA fragment containing 3HA tag into the NotI site which was introduced after ATG codon of HEM12 orf by PCR in vitro mutagenesis of pBS-HEM12 (Zoladek et al., 1995). Then SacI-HindIII fragment containing HA-HEM12 was transferred to SacI- and HindIII-digested pRS415 and pRS425 (Invitrogen) to obtain pRS415-HA-HEM12 and pRS425-HA-HEM12. Mutations in HEM12 were introduced by PCR mutagenesis of pBS-HA-HEM12, respective fragments transferred to pRS415 and pRS425 and confirmed by sequencing. Also, the SacI-HindIII fragment of pBS-HEM12 was used to obtain pRS415-HEM12.
Purification of ubiquitinated proteins. Purification of His-tagged ubiquitinated protein was performed as described previously (Stawiecka-Mirota et al., 2007). Total extracts, Ni-NTA-sepharose (Qiagen)-bound and unbound fractions were analyzed by Western blotting using anti-HA antibody.

Western blotting. Protein extracts were prepared and Western blotting was performed as described previously (Stawiecka-Mirota et al., 2007). Anti-Nedd4 WW domain recognizing Rsp5 (Millipore), anti-Pgk1 (Molecular Probes), monoclonal anti-HA (12CA5, Covance), monoclonal anti-Vma2 (Molecular Probes) primary antibodies and secondary horseradish peroxidase-conjugated antibodies (DACO) were used and followed by enhanced chemiluminescence (Millipore). HA-Hem12 and Rsp5 levels were quantified using Image Quant and normalized to Vma2, Pgk1 or Ponceau Red stained proteins.

Real-time PCR. Real-time RT-PCR was performed using the LightCycler ${ }^{\circledR} 480$ System (Roche Laboratories) with SYBR Green detection. The primers' specificity was verified by melting curve analysis. Sequences of primers used are available upon request. RT-PCRs were performed in triplicate. cDNA was synthesized from 5 $\mu \mathrm{g}$ of total RNA using RevertAid ${ }^{\mathrm{TM}} \mathrm{H}$ Minus M-MuLV Reverse Transcriptase kit (Fermentas). Average cycle thresholds were calculated and the Pfaffl method (Pfaffl, 2001) was used to calculate relative HEM12 expression levels with respect to $5 \mathrm{~S}$ rRNA.

Computational analysis. A protein multiple sequence alignment was obtained with the MAFT program (Katoh \& Toh, 2008) using homologs identified with BLAST (Schaffer et al., 2001). Several homologs of $S$. cerevisiae Hem12 with solved crystal structures have been indicated by BLAST and HHpred (Soding et al., 2005) servers. The structure of human uroporphyrinogen decarboxylase (PDB entry 1URO; (Whitby et al., 1998)) was chosen as a template to construct a model of Hem12 protein. Structural models of wild type and mutant Hem12 were obtained using the Sybyl-x1.2 package (TRIPOS, Inc., USA). The model structures were subjected to energy minimization using AMBERFF99 force field as implemented in Sybyl-x1.2.

\section{RESULTS AND DISCUSSION}

\section{The Hem 12 protein is monoubiquitinated by the Rsp5 ligase in vivo}

Since in vitro experiments had identified Hem12 as a substrate of Rsp5 (Gupta et al., 2007), we inquired if Rsp5 contributes to regulation of the heme biosynthesis pathway via in vivo ubiquitination and regulation of Hem12. To answer this question we used a HA-tagged version of HEM12 expressed from a plasmid restoring viability of a hem $12 \Delta$ strain on rich glucose medium at $28^{\circ} \mathrm{C}$ (Fig. 1A). The wild type or the $r s p 5 \Delta$ strain, where a plasmid encoding Spt23 $3^{1-689}$ ensured viability (Hoppe et al., 2000), were transformed with single- or multicopy plasmids bearing $H A$-HEM12. Western blot analysis revealed that the level of HA-Hem12 overexpressed from a multicopy plasmid was increased 13-16 fold (Fig. 1B). To test if Hem12 is ubiquitinated in vivo we used strains expressing HIS-UBI encoding His-ubiquitin (StawieckaMirota et al., 2007) and HA-HEM12 from the multicopy plasmid. His-ubiquitinated proteins were purified on Ni-sepharose beads and were analyzed by Western blotting with anti-HA antibody. Results shown in Fig. 1C document that HA-Hem12 is monoubiquitinated in vivo. 

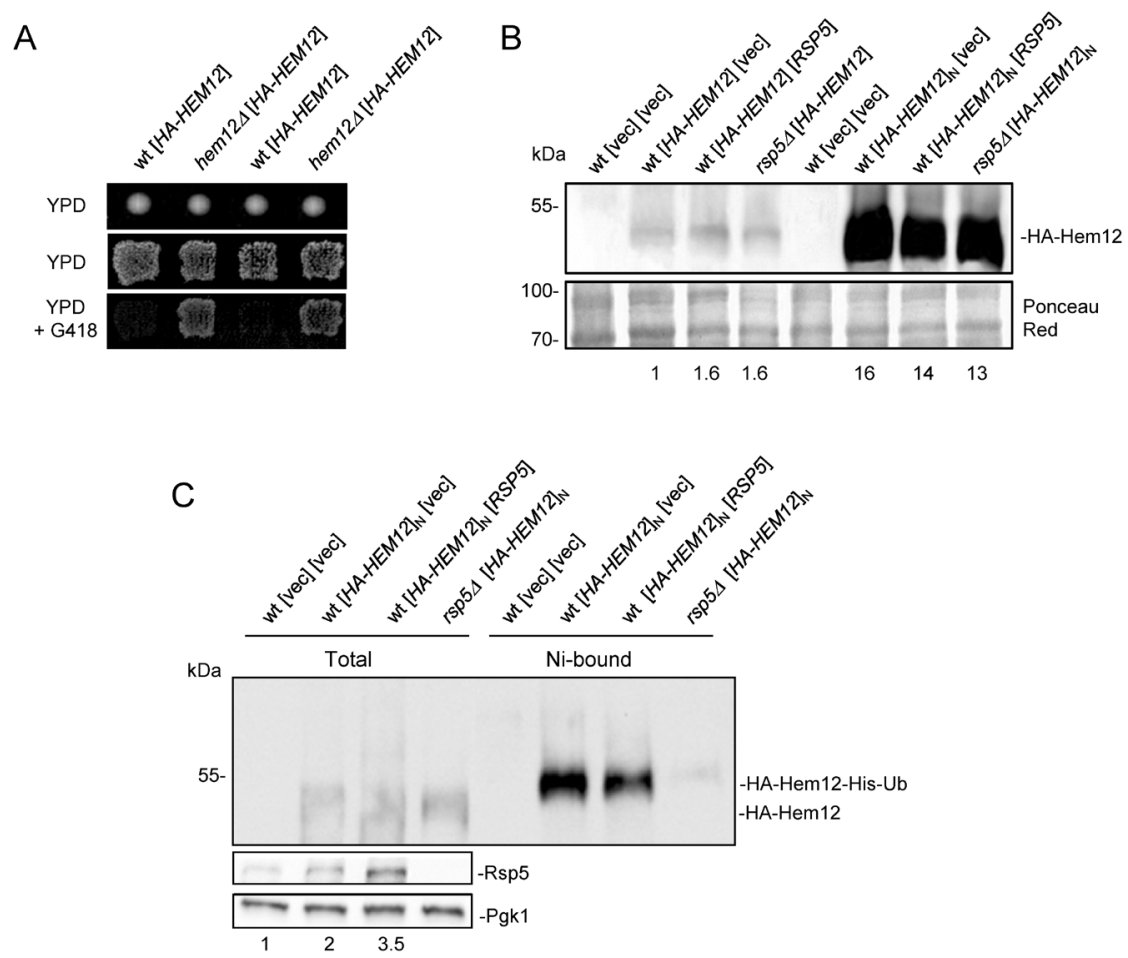

Figure 1. Hem 12 protein is monoubiuqitinated in vivo and this ubiquitination depends on Rsp5.

(A) HA-HEM 12 complements lethality of hem 12A. Diploid HEM12/hem12A::kanMX4 was transformed with pRS415-HA-HEM12 plasmid, tetrads were obtained and spore clones tested for growth on YPD and YPD+G418. (B) Additional copy of RSP5 or deletion of RSP5 does not affect the level of Hem12. Wild type strain (MHY501) was transformed with an empty vector, pRS415-HA-HEM12 or multicopy pRS425HA-HEM12 ([HA-HEM12] $]_{N}$ ) and with YCp33-RSP5 or empty vector for control. Strain rsp5 bearing plasmid expressing SPT231-689 was transformed with pRS415-HA-HEM12 or pRS425-HA-HEM12. All of these strains also contained the YEp-HIS-UBI plasmid. Protein extracts were analyzed by Western blotting with anti-HA antibody. (C) Hem 12 is ubiquitinated. Transformants expressing HIS-UBI and HA-HEM12 from multicopy plasmids as in B, were grown, protein extracts were prepared, and His-ubiquitinated proteins were purified using Ni-sepharose resins. Total extracts and Ni-bound fractions were analysed by Western blotting with anti-HA antibody. Rsp5 was detected by anti-Nedd4 antibody and anti-Pgk1 was used to control for protein loading. HA-Hem12 and Rsp5 levels were quantified.

When the Rsp5 ubiquitin ligase is absent, ubiquitination of Hem12 is abolished to nearly background levels, which may indicate that in the absence of Rsp5 another ligase partially takes over. An additional copy of RSP5 giving rise to a 3.5-fold elevated level of Rsp5 essentially did not increase the ubiquitination of Hem12. Thus, Rsp5 ubiquitinates Hem12 in vivo and some factors control the level of this ubiquitination tightly. The presence of an additional copy or deletion of RSP 5 essentially did not affect the steady state level of the Hem12 protein (Fig. 1B, C). Unlike polyubiquitination, monoubiquitination does not direct proteins to proteasomal degradation since proteasomes only recognize proteins tagged with K48-linked polyubiquitin chains containing more than four ubiquitins. Instead, monoubiquitination recruits proteins containing ubiquitin-binding domains (UBDs) and provides a signaling mechanism that regulates several cellular pathways (Ikeda \& Dikic, 2008). Thus, the Rsp5dependent monoubiquitination probably does not direct Hem12 for degradation but could affect its functioning through some other mechanism.

\section{K8A substitution in Hem12 affects growth of cells on glycerol medium at an elevated temperature}

Hem12 is highly conserved in evolution. UROD has a homodimeric structure with the active site clefts facing each other and all decarboxylation events occurring only in one active site (Martins et al., 2001; Bushnell et al., 2010). Assuming that the regulation of UROD by ubiquitination might be also conserved, we compared 34
UROD amino acid sequences to find conserved lysine residues which could be potential ubiquitination sites. Alignment of three sequences is presented in Fig. 2A. We found that five lysines: K8, K99, K174, K242 and K260 of Hem12 are conserved. Computer modeling of the Hem12 structure (Fig. 2B) showed that two of them, K174 and K260, are located on the dimer interface but three others are potentially available for ubiquitination. Since $\mathrm{K} 8$ is very close to the PY motif 36-LPEY-39 which binds the WW1 and WW3 domains of Rsp5 (Hesselberth et al., 2006) we assumed that this lysine is likely the ubiquitination site. Computer modeling indicated that substitution of $\mathrm{K} 8$ with alanine should not affect the protein structure (not shown). Therefore, the HA-hem 12 $K 8 A$ mutant allele was constructed by in vitro mutagenesis and tested for complementation of hem $12 \Delta$ inviability. A heterozygous HEM12/hem12A strain was transformed with a plasmid bearing the mutant allele, sporulated and tetrads were dissected. The hem12 $\Delta$ [HA-hem12-K8A] spores were viable and their growth was comparable to that of wt [HA-hem12-K8A] and hem12S [HA-HEM12] strains on medium containing glucose or glycerol, a nonfermentable carbon source, at $28^{\circ} \mathrm{C}$ (Figure $3 \mathrm{~A}$ and not shown). However, the hem12 $\triangle H A$-hem12-K8A] mutant strain grew significantly slower on the glycerol medium at $38^{\circ} \mathrm{C}$ when compared with wt [HA-hem12-K8A] and hem12 $\triangle$ [HA-HEM12] (Fig. 3A and not shown). We observed a similar steady state level of HA-Hem12 and HA-Hem12-K8A proteins in cells grown at $28^{\circ} \mathrm{C}$, and a similar 5-fold decrease of the steady state level after 4 hours of incubation at $38^{\circ} \mathrm{C}$ (Fig. 3B). These results 
A

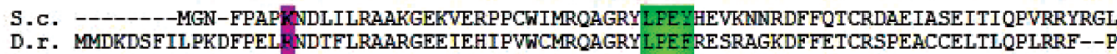
D.r. MEANGLGPQG-FPEL NDTFLRAAWGEETDYTPVWCMRQAGRYLPEERETRAAQDFFSTCRSPEACCELTLQPLRRF--I

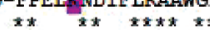

S.C. IDAAIIFSDILVI POAMGMRVEMLEG GKPHFPEPLRNPEDLOTVLDYKVDVLKELDWAFKAITMTRIKLDGEVPLFGFCG D.r. FDAAIIFSDILVVPQAMGMEVQMCPG GPTFPEPLKEPEDLQRLKT-QVDVYSELDYVFKAITLTRHKIEGKVPLIGFTG

H.S. LDAAIIFSDILVVPOALGMEVTMVPG GPSFPE PLREEQDLERLRD-PEVVASELGYVFOAITLTRQRLAGRVPLIGFAG

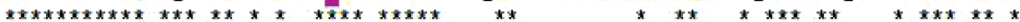

S.c. GPWTIMVYMTEGGGSRLFRFAF̄OWIMYYPELSHKLLQKITDVAVEFLSOOVVAGAOILOVFESWGGELSSVDFDEFSLPYI D.r. APWTLMS YMIEGGGSATHSKA RWLYRYPEASHKLLSOLTDVIVEYLLGQVKAGAOALQVFESHTGCLGPVEFKEFSLPYI

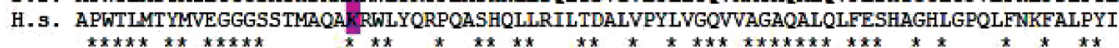

S.c. RQIAERVFKK-RLQELGIMEQI PMIVFAFGSWYALDKLCCSGFDVVSLDWSWDPREAVKINKARVTLQGNLDPGVMYGSEV D. $r$. RDIARRV-WDKIKESGL-DNVPMIVFA DGHYGLEDLSESAYEVVGLDWTIDPRSARVRTGGKVSLQGMMDPCALYGTKES H.s. RDVAKQV-KARLREAGL-APVPUIIFAKGGHFALEELAQAGYEVVGLDWTVAPKKARECVGKTVTLQGMLDPCALYASEEE

S.c. ITKKVKOMIEAFGGGKSRYIVNFGHGTHPFMDPDVIKFFLEECHRIG-SK---

D. $r$. ISEIVRRMLEGF--GTKGYIANLGHGLYPDMDPENVGAFVEAVHNHSROLIKR

H.s. IGQLVKQMLDDF--GPHRYIANLGHGLYPDMDPEHVGAFVDAVHKHSRLLRQN

B

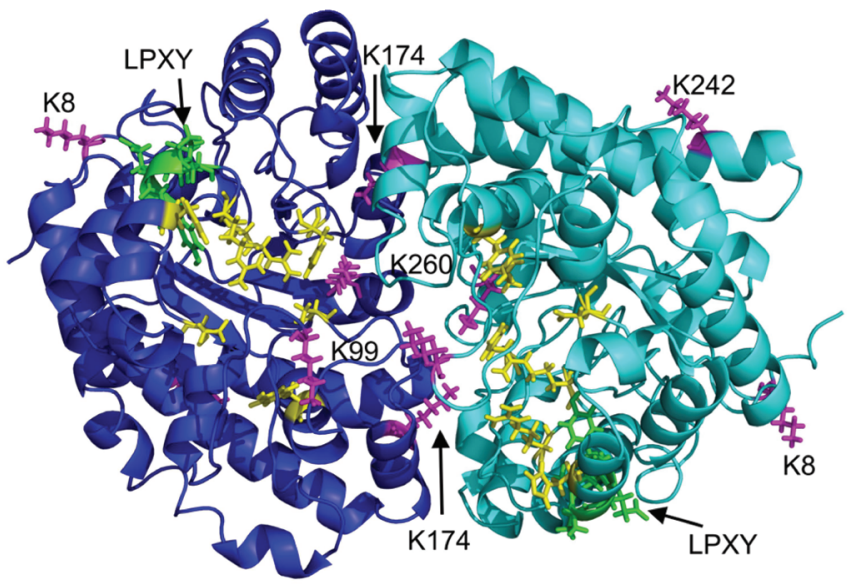

Figure 2. Five lysines are conserved in UROD.

(A) Alignment of amino acid sequences of UROD from S. cerevisiae, Danio rerio and Homo sapiens. Evolutionarily conserved lysines are purple, LPEY/F motif is in green. (B) Model of S. cerevisiae Hem12 structure. The active enzyme is a homodimer, individual monomers are shown in light and dark blue. Amino acid residues forming the active site are marked in yellow. Conserved lysines K8, K99, K174, K242 and K260 are in pink. Conserved LPEY motif which binds WW domains of Rsp5 is in green.

indicate that Hem12 is subject to downregulation upon heat stress and suggest that the bem12-K8A mutation does not affect the stability of Hem12 but negatively affects its activity or interaction with other proteins at the elevated temperature. Lysines other than K8 of Hem 12 could be ubiquitinated and direct the wild type and mutant Hem12-K8A protein for degradation at an elevated temperature. In fact, Hem12-K8A mutant protein is still monoubiquitinated (not shown).

To investigate if Rsp5 is important for the degradation of Hem12 upon heat stress, the level of wild type Hem12 was monitored in wild type yeast and rsp5-1 mutant with the L733S substitution in the catalytic HECT domain showing a temperature-sensitive growth defect (Wang et al., 2001). Yeast were grown at $28^{\circ} \mathrm{C}$, shifted for 2 or 4 hours to $38^{\circ} \mathrm{C}$, and Western blotting was performed. The steady state level of Hem12 was lower in rsp5-1 than in wild type cells but this protein was similarly degraded upon heat stress in both strains showing again that Rsp5 does not affect its degradation (Fig. 3C). This is in contrast to the recent finding that Rsp5 is the main ubiquitin ligase that targets cytosolic proteins following heat stress (Fang et al., 2014). Thus, another ligase must be involved in forming polyubiquitin chains that direct Hem12 for proteasomal degradation. Previous in vitro experiments have revealed that Hem12 may be also a substrate of the cytosolic ligase Ubr1 ( $\mathrm{Lu}$ et al.,
2008) which functions in quality control of cytoplasmic proteins (Eisele \& Wolf, 2008; Xia et al., 2008). It is possible that Rsp5 and Ubr1 function together in Hem12 ubiquitination in vivo. Moreover, Ubr1 could be responsible for the residual monoubiquitination of Hem12 observed in the $r s p 5 \Delta$ strain.

\section{Double mutation hem 12-L36A,P37A destroying the PY motif in Hem12 prevents cell growth on glycerol medium}

To eliminate Rsp5 binding and ubiquitination of Hem12, L36A and P37A mutations which make the PY motif not functional (Merhi \& Andre, 2012) were introduced into a plasmid carrying HA-HEM12. Computer modeling showed that these mutations should not affect the structure of the Hem12 catalytic center (not shown) and thus should not affect its catalytic activity. The heterozygous HEM12/hem12A strain was transformed with the above plasmid, sporulated and spore clones were isolated. The hem12 $[H A$-hem12-L36A,P37A] spores were viable but grew extremely slowly at $28^{\circ} \mathrm{C}$ on the glucose medium and not at all on the glycerol medium (Fig. 4A) suggesting that the mutant protein is barely active. This phenotype is much stronger than phenotype of bem12-K8A mutant suggesting that another lysine could be ubiquitinated when $\mathrm{K} 8$ is absent. Interestingly, after 2-3 passages on the glucose medium hem $12 \Delta$ 
A

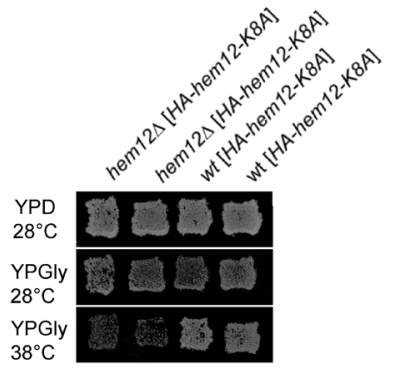

B

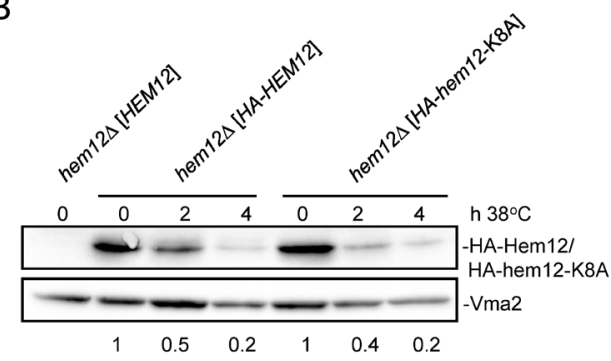

C

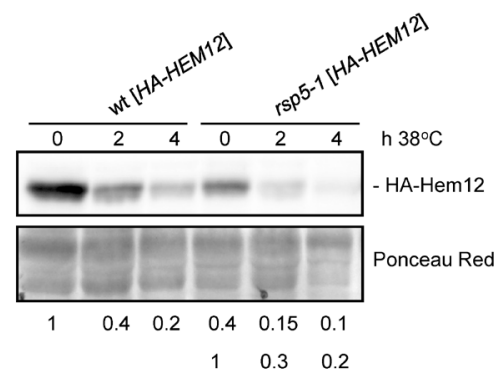

Figure 3. Effect of hem12-K8A mutation on cell growth on glycerol medium and the level of mutant HA-Hem12 protein.

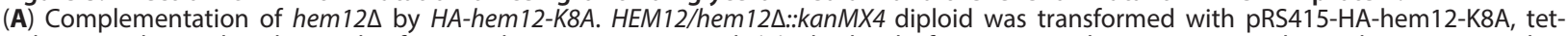
rads were obtained and growth of spore clones was compared. (B) The level of HA-Hem12 decreases at an elevated temperature but is not affected by hem12-K8A mutation. The hem12 [HEM12], hem12 $[H A-H E M 12]$ and hem12 $[H A-h e m 12-K 8 A]$ strains were grown in YPGly at $28^{\circ} \mathrm{C}$, shifted or not shifted to $38^{\circ} \mathrm{C}$ for 2 or 4 hours, extracts were analyzed by Western blotting using anti-HA and anti-Vma2 antibodies. (C) The rsp5-1 mutation does not affect the degradation of Hem12 at an elevated temperature. Western blot using anti-HA antibody of wild type and rsp5-1 cells expressing HA-Hem12 from single copy plasmid. HA-Hem12 levels were quantified.

A

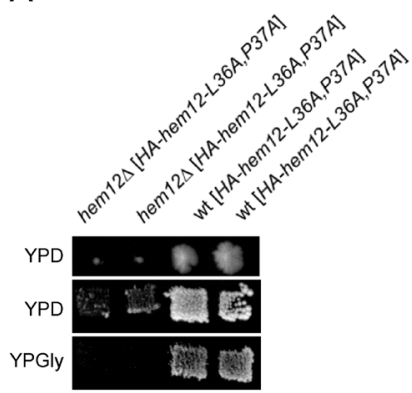

C

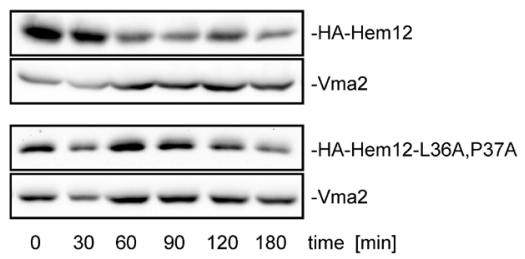

B
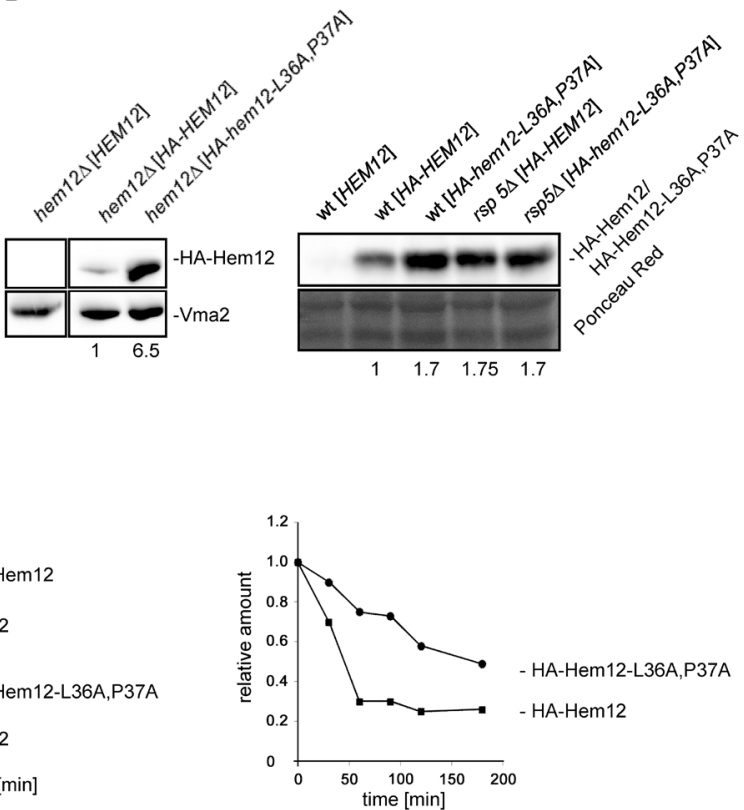

Figure 4. Effect of hem12-L36A,P37A mutation on cell growth and the level and stability of mutant Hem12 protein.

(A) Complementation of hem12L by HA-hem12-L36A,P37A. HEM12/hem12L::kanMX4 diploid was transformed with pRS415-HA-hem12L36A,P37A, tetrads were obtained and growth of spore clones was tested on YPD and YPGly at $28^{\circ} \mathrm{C}$. (B) The level of HA-Hem12-

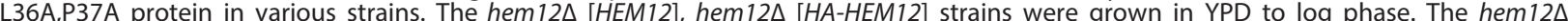
[HA-hem12- L36A,P37A] strain was reinoculated three times into fresh YPD and then grown to log phase. Extracts were analyzed by Western blotting using anti-HA and anti-Vma2 antibodies (left panel). The wt [HA-HEM12], wt [HA-hem 12- L36A,P37A], rsp5 [HA-HEM12] and rsp5 $[$ [HA-hem12- L36A,P37A] strains were grown in SC medium, transferred to YPD and allowed to grow for two generations. Extracts were analyzed using anti-HA antibodies (right panel). One representative experiment of two is shown. (C) Cycloheximide chase analysis of wild type and mutant Hem12 levels in hem 12 [HA-HEM12] and hem12 [HA-hem12- L36A,P37A] strains. HA-Hem12 turnover was monitored by Western blotting using anti-HA and anti-Vma2 antibodies at indicated chase times after addition of cycloheximide to cultures grown in YPD medium. HA-Hem12 levels were quantified. 
[HA-bem12-L36A,P37A] spore clones achieved a normal rate of growth. Western blotting showed that the level of the mutant Hem12-L36A,P37A was greatly elevated in those strains, up to 6.5-fold over the wild type level (Fig. 4B, left panel). The glucose-adapted hem12 [HA-bem12-L36A,P37A] spore clones also grew on the glycerol medium (not shown). The level of HA-Hem12L36A,P37A in wild type and $r s p 5 \Delta$ strain transformed with a plasmid bearing $H A$-hem 12-L36A,P37A gene was similar to that of the wild type HA-Hem12 protein (Fig. 4B, right panel). This shows that HA-Hem12L36A,P37A is present at increased levels only in the absence of wild type Hem 12 protein and also that Rsp 5 is not involved in its degradation.

The high steady state level of HA-Hem12-L36A,P37A protein in hem12A [HA-hem12-L36A,P37A] cells could result from an increased stability of the protein, from the induction of $H A$-hem 12-L36A,P37A expression, or both. However, in our previous experiments (Fig. 1B) deletion of the RSP5 gene did not significantly affect the level of HA-Hem12, suggesting that the mutation of Hem 12 preventing its modification by Rsp 5 should not have caused its stabilization. To clarify that, Hem12 stability was investigated. Cycloheximide was added to logarithmic phase cultures of wild type and hem $12 \Delta$ [HA-bem12-L36A,P37A] spore clones to inhibit cytosolic protein synthesis and cell samples were taken at 30 or 60-min intervals. Western blot analysis of cell extracts showed that in fresh spore clones the level of mutant HA-Hem12-L36A,P37A was lower compared with that of the wild type (Fig. 4C, left panel time 0). The low level of the mutant protein cannot be solely responsible for growth defect of hem12A [HA-hem12-L36A,P37A] spore clones since the $\mathrm{Sm} 39$ mutant showing a similar low level of wild type Hem12 grows well on glycerol medium (Zoladek et al., 1995). The wild type Hem12 protein was rather stable and was degraded in two phases, a faster one with a half-life below $50 \mathrm{~min}$ and a slower phase with a half-life above $200 \mathrm{~min}$ (Fig. 4C, right panel). This result indicates that two pools of Hem12 protein are present in wild type cells, one that is prone to degradation and one protected from degradation, possibly by binding to other proteins. Degradation of the mutant HA-Hem12-L36A,P37A was slower than that of wild type Hem12, especially in the first phase. Thus, preventing the binding of Hem 12 by Rsp 5 resulted in Hem 12 stabilization. Considering that Rsp5 does not affect the degradation of Hem 12 this effect is probably indirect and unrelated to Hem12 ubiquitination by Rsp5.

To learn if changes in expression of the HEM12 gene contribute to the observed high level of HA-Hem12L36A,P37A mutant protein, the HEM12 transcript was analyzed. RNA was isolated from wild type and bem12A [HA-hem12-L36A,P37A] spore clones propagated on glucose medium for three days, and quantified by RT-PCR. The level of $H A$-hem 12-L 36A,P37A mRNA was 2.15 fold higher than that of HEM12 mRNA. These results collectively show that the HA-hem12-L36A,P37A mutant does not initially grow on the glycerol medium possibly because of low activity of the mutant HA-Hem12L36A,P37A protein but easily adapts to that medium by increasing the level of the mutant protein through increased expression of the encoding gene and increased stability of the mutant HA-Hem12-L36A,P37A protein.

The obtained results led us to propose a model in which Hem12 is bound and mono-ubiquitinated by Rsp5, possibly at K8. The Rsp5-dependent ubiquitination does not direct Hem12 to degradation. The Rsp5 binding and the monoubiquitination of Hem12 indepen- dently help it to bind some unidentified protein which is required for efficient heme synthesis, cell survival and ability to grow on the glycerol medium.

Yeast Hem12 protein shows 50\% identity and 67\% similarity to the human UROD enzyme, which contains the LPEF motif. Therefore, human UROD could be also ubiquitinated and regulated by a human ubiquitin ligase of the Nedd4 family. Mutations affecting monoubiquitination or promoting degradation of UROD could possibly be one of the reasons of PCT in humans for which no mutation in the UROD gene has been found. Further work will be required to confirm the postulated ubiquitination of UROD and to establish its physiological role in humans.

\section{Acknowledgments}

This work was supported by grant UMO-2011/03/B/ NZ1/00570 from the National Science Centre, Poland. T.Z. lab is a member of COST Action BM1307 PROTEOSTASIS.

\section{REFERENCES}

Bushnell EA, Erdtman E, Llano J, Eriksson LA, Gauld JW (2011) The first branching point in porphyrin biosynthesis: a systematic docking, molecular dynamics and quantum mechanical/molecular mechanical study of substrate binding and mechanism of uroporphyrinogen-III decarboxylase. J Comput Chem 32: 822-834. http:// dx.doi.org/10.1002/jcc.21661.

Chen P, Johnson P, Sommer T, Jentsch S, Hochstrasser M (1993) Multiple ubiquitin-conjugating enzymes participate in the in vivo degradation of the yeast MAT alpha 2 repressor. Cell 74: 357-369.

Diflumeri C, Larocque R, Keng T (1993) Molecular analysis of HEM6 (HEM12) in Saccharomyces cerevisiae, the gene for uroporphyrinogen decarboxylase. Yeast 9: 613-623.

Eisele F, Wolf DH (2008) Degradation of misfolded protein in the cytoplasm is mediated by the ubiquitin ligase Ubr1. FEBS Lett 582: 4143-4146. http://dx.doi.org/10.1016/j.febslet.2008.11.015.

Fang NN, Chan GT, Zhu M, Comyn SA, Persaud A, Deshaies RJ, Rotin D, Gsponer J, Mayor T (2014) Rsp5/Nedd4 is the main ubiquitin ligase that targets cytosolic misfolded proteins following heat stress. Nat Cell Biol 16: 1227-1237. http://dx.doi.org/10.1038/ ncb3054.

Frank J, Poblete-Gutierrez P (2010) Porphyria cutanea tarda--when skin meets liver. Best Pract Res Clin Gastroenterol 24: 735-745. http:// dx.doi.org/10.1016/j.bpg.2010.07.002.

Garey JR, Franklin KF, Brown DA, Harrison LM, Metcalf KM, Kushner JP (1993) Analysis of uroporphyrinogen decarboxylase complementary DNAs in sporadic porphyria cutanea tarda. Gastroenterology 105: 165-169.

Gupta R, Kus B, Fladd C, Wasmuth J, Tonikian R, Sidhu S, Krogan NJ, Parkinson J, Rotin D (2007) Ubiquitination screen using protein microarrays for comprehensive identification of Rsp5 substrates in yeast. Mol Syst Biol 3: 116.

Harreman M, Taschner M, Sigurdsson S, Anindya R, Reid J, Somesh B, Kong SE, Banks CA, Conaway RC, Conaway JW, Svejstrup JQ (2009) Distinct ubiquitin ligases act sequentially for RNA polymerase II polyubiquitylation. Proc Natl Acad Sci U S A 106: 2070520710. http://dx.doi.org/10.1073/pnas.0907052106.

Heinemann IU, Jahn M, Jahn D (2008) The biochemistry of heme biosynthesis. Arch Biochem Biophys 474: 238-251. http://dx.doi.org/10.1016/j.abb.2008.02.015.

Hesselberth JR, Miller JP, Golob A, Stajich JE, Michaud GA, Fields S (2006) Comparative analysis of Saccharomyces cerevisiae WW domains and their interacting proteins. Genome Biol 7: R30.

Hoffman M, Gora M, Rytka J (2003) Identification of rate-limiting steps in yeast heme biosynthesis. Biochem Biophys Res Commun 310: $1247-1253$

Hoppe T, Matuschewski K, Rape M, Schlenker S, Ulrich HD, Jentsch S (2000) Activation of a membrane-bound transcription factor by regulated ubiquitin/proteasome-dependent processing. Cell 102: 577-586.

Hoshikawa C, Shichiri M, Nakamori S, Takagi H (2003) A nonconserved Ala401 in the yeast Rsp5 ubiquitin ligase is involved in degradation of Gap1 permease and stress-induced abnormal proteins. Proc Natl Acad Sci U S A 100: 11505-11510.

Ikeda F, Dikic I (2008) Atypical ubiquitin chains: new molecular signals. 'Protein Modifications: Beyond the Usual Suspects' review 
series. EMBO Rep 9: 536-542. http://dx.doi.org/ 10.1038/embor.2008.93.

Kaliszewski P, Zoladek T (2008) The role of Rsp5 ubiquitin ligase in regulation of diverse processes in yeast cells. Acta Biochim Pol 55: 649-662.

Katoh K, Toh H (2008) Recent developments in the MAFFT multiple sequence alignment program. Brief Bioinform 9: 286-298. http:// dx.doi.org/10.1093/bib/bbn013.

Kee Y, Munoz W, Lyon N, Huibregtse JM (2006) The deubiquitinating enzyme Ubp2 modulates Rsp5-dependent Lys63-linked polyubiquitin conjugates in Saccharomyces cerevisiae. I Biol Chem 281: 36724-36731.

Kurlandzka A, Zoladek T, Rytka J, Labbe-Bois R, Urban-Grimal D (1988) The effects in vivo of mutationally modified uroporphyrinogen decarboxylase in different hem12 mutants of baker's yeast ( $\mathrm{Sac}$ charomyces cerevisiae). Biochem I 253: 109-116.

Lauwers E, Erpapazoglou Z, Haguenauer-Tsapis R, Andre B (2010) The ubiquitin code of yeast permease trafficking. Trends Cell Biol 20: 196-204. http://dx.doi.org/10.1016/j.tcb.2010.01.004.

Lu JY, Lin YY, Qian J, Tao SC, Zhu J, Pickart C, Zhu H (2008) Functional dissection of a HECT ubiquitin E3 ligase. Mol Cell Proteomics 7: $35-45$.

Martins BM, Grimm B, Mock HP, Huber R, Messerschmidt A (2001) Crystal structure and substrate binding modeling of the uroporphyrinogen-III decarboxylase from Nicotiana tabacum. Implications for the catalytic mechanism. I Biol Chem 276: 44108-44116.

Mendez M, Rossetti MV, Gomez-Abecia S, Moran-Jimenez MJ, Parera V, Batlle A, Enriquez de Salamanca R (2012) Molecular analysis of the UROD gene in 17 Argentinean patients with familial porphyria cutanea tarda: characterization of four novel mutations. Mol Genet Metab 105: 629-633. http://dx.doi.org/10.1016/j. ymgme.2012.02.002.

Merhi A, Andre B (2012) Internal amino acids promote Gap1 permease ubiquitylation via TORC1/Npr1/14-3-3-dependent control of the Bul arrestin-like adaptors. Mol Cell Biol 32: 4510-4522. http://dx.doi.org/10.1128/MCB.00463-12.

Novoselova TV, Rose RS, Marks HM, Sullivan JA (2013) SUMOylation regulates the homologous to E6-AP carboxyl terminus (HECT) ubiquitin ligase Rsp5p. J Biol Chem 288: 10308-10317. http://dx.doi. org $/ 10.1074$ /ibc.M112.424234.

Panda S, Antoch MP, Miller BH, Su AI, Schook AB, Straume M, Schultz PG, Kay SA, Takahashi JS, Hogenesch JB (2002) Coordinated transcription of key pathways in the mouse by the circadian clock. Cell 109: 307-320.
Pfaffl MW (2001) A new mathematical model for relative quantification in real-time RT-PCR. Nucleic Acids Res 29: e45.

Schaffer AA, Aravind L, Madden TL, Shavirin S, Spouge JL, Wolf YI, Koonin EV, Altschul SF (2001) Improving the accuracy of PSIBLAST protein database searches with composition-based statistics and other refinements. Nucleic Acids Res 29: 2994-3005.

Sherman F (2002) Getting started with yeast. Methods Enzymol 350: $3-41$.

Soding J, Biegert A, Lupas AN (2005) The HHpred interactive server for protein homology detection and structure prediction. Nucleic Acids Res 33: W244-248.

Stawiecka-Mirota M, Pokrzywa W, Morvan J, Zoladek T, Haguenauer-Tsapis R, Urban-Grimal D, Morsomme P (2007) Targeting of Sna3p to the endosomal pathway depends on its interaction with Rsp5p and multivesicular body sorting on its ubiquitylation. Traffic 8: 1280-1296.

Wang G, McCaffery JM, Wendland B, Dupre S, Haguenauer-Tsapis R, Huibregtse JM (2001) Localization of the Rsp5p ubiquitin-protein ligase at multiple sites within the endocytic pathway. Mol Cell Biol 21: $3564-3575$.

Whitby FG, Phillips JD, Kushner JP, Hill CP (1998) Crystal structure of human uroporphyrinogen decarboxylase. Embo J 17: 2463-2471.

Wu N, Yin L, Hanniman EA, Joshi S, Lazar MA (2009) Negative feedback maintenance of heme homeostasis by its receptor, Reverbalpha. Genes Dev 23: 2201-2209. http://dx.doi.org/10.1101/ gad.1825809.

Xia Z, Webster A, Du F, Piatkov K, Ghislain M, Varshavsky A (2008) Substrate-binding sites of UBR1, the ubiquitin ligase of the Nend rule pathway. I Biol Chem 283: 24011-24028. http://dx.doi. org/10.1074/jbc.M802583200.

Yip KW, Zhang Z, Sakemura-Nakatsugawa N, Huang JW, Vu NM, Chiang YK, Lin CL, Kwan JY, Yue S, Jitkova Y, To T, Zahedi P, Pai EF, Schimmer AD, Lovell JF, Sessler JL, Liu FF (2014) A porphodimethene chemical inhibitor of uroporphyrinogen decarboxylase. PLoS One 9: e89889. http://dx.doi.org/10.1371/journal. pone.0089889.

Zoladek T, Chelstowska A, Labbe-Bois R, Rytka J (1995) Isolation and characterization of extragenic mutations affecting the expression of the uroporphyrinogen decarboxylase gene (HEM12) in Sacharomyces cerevisiae. Mol Gen Genet 247: 471-481.

Zoladek T, Nguyen BN, Rytka J (1996) Saccharomyces cerevisiae mutants defective in heme biosynthesis as a tool for studying the mechanism of phototoxicity of porphyrins. Photochem Photobiol 64: 957-962. 